\title{
Bimodules and Rota-Baxter Relations
}

\section{Ibrahima Bakayoko ${ }^{1 *}$ and Momo Banagoura ${ }^{2}$}

${ }^{1}$ Departement' of Mathematics, UJNK/N'Zerekore University Centre, BP : 50, NZerekore Guinea University of Maryland College Park, MD 20742, USA

${ }^{2}$ Departement of Mathematics, University Gamal Abdel Nasser of Conakry, BP 1147, Conakry, Guinea

\begin{abstract}
In this paper we introduce and study Hom-type bimodules of some Hom-algebraic structures endowed with Rota-Baxter relations. We introduce bimodules over Homassociative Rota-Baxter algebras and give their various twisting and their connection with bimodules over Hom-preLie algebras. Then we introduce Rota-Baxter q- Homtridendriform algebras. Next we express axioms defining q-Hom-tridendriform algebras by mean of vector basis. Moreover we introduce bimodules over q-Homtridendriform algebras and give some examples, and prove that they are closed by twisting. Finally we give their connection with Hom-associative Rota-Baxter bimodules.
\end{abstract}

Keywords: Hom-preLie; Q-Hom-tridendriform and Homassociative Rota-Baxter algebras; Rota-Baxter bimodules

AMS Subject Classification: 16W20, 17D25, 16T15

\section{Introduction}

Rota-Baxter operators have appeared in a wide range of areas in pure and applied mathematics. The paradigmatic example of a RotaBaxter operator concerns the integration by parts formula. RotaBaxter algebras find their application in probability and the study of fluctuation theory [1], combinatorics [2,3], quantum field theory [4], Lie algebra theory $[5,6]$. It is shown that the Rota-Baxter identity can be significant in the construc-tion of Frobenius manifolds inherent to the integrable systems of hydrodynamic type [7]. They are closly related to dendriform algebra [8] which were introduced by Loday. Dendriform algebras have two binary operations, which dichotomize an associative multiplication. The motivation to introduce these algebraic structures comes from K-theory. Dendriform algebras are connected to several areas in mathematics and physics, including Hopf algebras, homotopy Gerstenhaber algebra, operads, homology, combinatorics, and quantum field theory, where they occur in the theory of renormalization of Connes and Kreimer. Rota-Baxter algebras are related to dendriform algebras via a pair of adjoint functors $[9,10]$. Roughly speaking, RotaBaxter algebras are to dendriform algebras as associative algebras are to Lie algebras. q-tridendriform algebras are introduced [11] as an algebraic structure with three operations containing dendriform algebras and tridendriform algebras as particular cases. The author studied tridendriform algebra structures of the space of parking fonctions and and of the space of multipermutations. Tridendriform bialgebras was also studied by the author.

Hom-algebra structures first arose in quasi-deformations of Lie algebras of vector fields. Discrete modifications of vector fields via twisted derivations lead to Hom-Lie and quasi-Hom-Lie structures, in which the Jacobi condition is twisted. Other interesting Hom-type algebraic structures of many classical structures were studied as Homassociative algebras, Hom-Lie admissible algebras and more general G-Hom-associative algebras [12], n-ary Hom-Nambu-Lie algebras [13], Hom-Lie admissible Hom-coalgebras and Hom-Hopf algebras [14], Hom-alternative algebras, Hom-Malcev algebras and HomJordan algebras [15], L-modules, L-comodules and Hom-Lie quasibialgebras [16], Laplacian of Hom-Lie quasi-bialgebras [17].

Hom-algebraic structures were extended to the case of G-graded Lie algebras by study-ing Hom-Lie superalgebras, Hom-Lie admissible superalgebras [18], color Hom-Lie al-gebras [19], color Hom-Lie bialgebras and color Hom-Poisson bialgebras [20] and color HomPoisson algebras. Color Hom-Poisson algebras were introduced [21] as generalization of Hom-Poisson algebras [12]. The generalized leftHom-symmetric algebras and generalized Hom-dendriform algebras as well as the corresponding modules are studied [22]. It is also proved that any generalized Hom-dialgebras give rise to generalized HomLeibniz-Poisson algebras and generalized Hom-Poisson dialgebras.

The aim of this paper is to introduce bimodules over some Homalgebraic structures endowed with Rota-Baxter relations. The paper is organized as follows. In section one, we recall basic notions related to modules over Hom-associative and Hom-Lie algebras. Section two is devoted to Rota-Baxter bimodules over Hom-associative Rota-Baxter algebras and their connection with bimodules over Hom-preLie algebras. In section three we introduce q-Hom-tridendriform RotaBaxter algebras. Most of the results (the varoius twisting) on q-Homtridendriform algebras are obviously analogs for Hom-tridendriform algebras and the proofs are identical nearly, so we omit them. We express axioms difining q-Hom-tridendriform algebras by mean of vector basis, which may be very usefull in the classification setting. In section four introduce bimodules theory for q-Hom-tridendriform algebra and we prove the commutativity of the following diagram

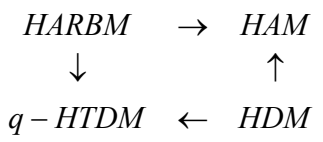

where HARBM denote the category of Hom-associative Rota-Baxter bimodules, q-HTDM denote the category of q-Hom-tridendriform bimodules, HDM denote the category of Hom-dendriform bimodules and HAM denote the category of Hom-associative bimodules.

All vector spaces considered are supposed to be over fields of characteristics different from 2 .

*Corresponding author: Ibrahima Bakayoko, Departement' of Mathematics, UJNK/N'Zerekore University Centre, BP: 50, NZerekore Guinea University of Maryland College Park, MD 20742, USA, Tel: 00224662046 669; E-mail: ibrahimabakayoko@yahoo.fr

Received July 24, 2015; Accepted August 18, 2015; Published August 29, 2015

Citation: Bakayoko I, Banagoura M (2015) Bimodules and Rota-Baxter Relations. J Appl Mech Eng 4: 178. doi:10.4172/2168-9873.1000178

Copyright: (c) 2015 Bakayoko I, et al. This is an open-access article distributed under the terms of the Creative Commons Attribution License, which permits unrestricted use, distribution, and reproduction in any medium, provided the original author and source are credited. 


\section{Preliminaries}

In this section, we recall basic definitions of Rota-Baxter Homassociative algebras and modules over Hom-Lie algebras.

Definition 1.1: [23] A Hom-associative algebra is a triple $(A, \bullet, \alpha)$ consisting of a linear space $\mathrm{A}$, a K-bilinear map $: A \times A \rightarrow A$ and a linear space map $\alpha: A \rightarrow A$ satisfying

$\alpha(x) \cdot(y \cdot z)=(x \cdot y) \cdot \alpha(z) \quad($ Hom-associativity)

If in addition $\alpha$ satisfies

$\alpha(x \cdot y)=\alpha(x) \cdot \alpha(y) \quad$ (multiplicativity),

then $(A, \bullet, \alpha)$ is said to be multiplicative.

When $\alpha=I d_{A},\left(A, \bullet I d_{A}\right)$, simply denoted $(A, \bullet)$, is an associative algebra.

Example 1.1: Let $\left\{e_{1}, e_{2}, e_{3}\right\}$ be a basis of a 3-dimensional vector space A over $\mathbf{K}$. The following multiplication $\bullet$ and map on A define a Hom-associative algebra [24]:

$$
\begin{aligned}
& e_{1} \cdot e_{1}=e_{1}, \quad e_{1} \cdot e_{2}=e_{2} \cdot e_{1}=e_{3} . \\
& \alpha\left(e_{1}\right)=a_{1} e_{2}+a_{2} e_{3}, \quad \alpha\left(e_{2}\right)=b_{1} e_{2}+b_{2} e_{3}, \quad \alpha\left(e_{3}\right)=c_{1} e_{2}+c_{2} e_{3} .
\end{aligned}
$$

Where $a_{1}, a_{2}, b_{1}, b_{2}, c_{1}, c_{2}$ are parameters in $\mathbf{K}$.

Definition 1.2: A Hom-associative Rota-Baxter algebra of weight $\lambda \in \mathbf{K}$ is a Hom-associative algebra $(A, \bullet, \alpha)$ together with a linear selfmap $R: A \rightarrow A$ that satisfies the identity[25]

$$
\begin{aligned}
& \alpha^{\circ} R=R^{\circ} \alpha, \\
& R(x) \cdot R(y)=R(x \cdot R(y)+R(x) \cdot y+\lambda x \cdot y) .
\end{aligned}
$$

If in addition commutes with $\bullet$, we say that $(A, \bullet, \alpha, R)$ is a multiplicative Hom-associative Rota-Baxter algebra of weight $\lambda$.

Definition 1.3: A Hom-Lie algebra is a triple $(L,[\bullet, \bullet], \alpha)$ consisting

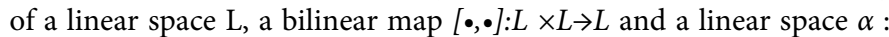
$L \rightarrow L$ satisfying [26]

$$
[x, y]=-[y, x] \quad \text { (skew-symmetry) }
$$

$[\alpha(x),[y, z]]+[\alpha(y),[z, x]]+[\alpha(z),[x, y]]=0 \quad$ (Hom-Jacobi identity)

When $\alpha: I d_{L}$, we obtain the definition of Lie algebras.

Lemma 1.1: Let $(A, \bullet, \alpha, R)$ be a multiplicative Hom-associative Rota-Baxter algebra of weight $\lambda$ and $\beta: A \rightarrow A$ be a morphism. Then $A_{\beta}=\left(A, \cdot \beta=\beta^{\circ}, \beta^{\circ} \alpha, R\right)$ is also a multiplicative Hom-associative RotaBaxter algebra of weight $\lambda[25]$.

We have a similar conclusion for Hom-Lie algebras.

Now we define modules over Hom-associative and Hom-Lie algebras.

Definition 1.4: A Hom-module is a pair $(M, \beta)$ in which $M$ is a $\mathbf{K}$-vector space and $\beta: M \rightarrow M$ is a linear map [27].

Definition 1.5: Let $(A, \bullet, \alpha)$ be a Hom-associative algebra and $(M, \beta)$ be a Hom-module. $A$ (left) $A$-module structure on $M$ consists of a K-bilinear $\succ: A \otimes M \rightarrow M$ such that [28]

$$
\begin{aligned}
& \beta(x \succ m)=\alpha(x) \succ \beta(m) \\
& \alpha(x) \succ(y \succ m)=(x \cdot y) \succ \beta(m)
\end{aligned}
$$

Definition 1.6: Let $(L,[\bullet, \bullet], \alpha)$ be a Hom-Lie algebra and $(M, \beta)$ be a Hom-module. An L-module on M consists of a K-bilinear $\succ: L \otimes M \rightarrow M$ such that for any $m \in M, x, y \in L,[27]$

$$
\begin{aligned}
& \beta(x \succ m)=\alpha(x) \succ \beta(m) . \\
& {[x, y] \succ \beta(m)=\alpha(x) \succ(y \succ m)-\alpha(y) \succ(x \succ m)}
\end{aligned}
$$
[19].

When $\beta: I d_{M}$ and $\alpha: I d_{L}$, we recover the definition of Lie modules

The following result shows that A-modules extend to L(A)-modules for the same module structure map.

Lemma 1.2: Let $(A, \bullet, \alpha)$ be a Hom-associative algebra and $(M, \succ, \beta)$ be an A-module. Then $\mathrm{M}$ is an $\mathrm{L}(\mathrm{A})$-module for the structure map $\succ \cdot[16]$

\section{Bimodules Over Hom-associative Rota-Baxter and Hom-pre Lie algebras}

In this section we introduce bimodules over Hom-associative RotaBaxter algebras. We give their various twisting and their connection with bimodules over Hom-preLie algebras.

Definition 2.1: Let $(A, \bullet, \alpha, R)$ be a Hom-associative Rota-Baxter algebra of weight and let $(M, \beta)$ be a Hom-module. A Hom-associative Rota-Baxter bimodule structure on $\mathrm{M}$ consists of:

A left A-action $\succ: A \otimes M \rightarrow M(x \otimes m \mapsto x \succ m)$,

Aright A-action, $\prec: M \otimes A \rightarrow M(m \otimes x \mapsto m \prec x)$, and

A Rota-Baxter operator $R_{M}: M \rightarrow M$ of weight $\lambda$

such that the following conditions hold for $x, y \in A$ and $m \in M$

$$
\begin{aligned}
& \beta(x \succ m)=\alpha(x) \succ \beta(m), \\
& \beta(m \prec x)=\beta(m) \prec \alpha(x), \\
& \alpha(x) \succ(y \succ m)=(x \cdot y) \succ \beta(m), \\
& (m \succ x) \prec \alpha(y)=\beta(m) \prec(x \cdot y), \\
& \alpha(x) \succ(m \prec y)=(x \succ m) \prec \alpha(y), \\
& \text { and } \\
& R_{M}{ }^{\circ} \beta=\beta^{\circ} R_{M} \\
& R(x) \succ R_{M}(m)=R_{M}\left(R(x) \succ m+x \succ R_{M}(m)+\lambda x \succ m\right), \\
& R_{M}(m) \prec R(x)=R_{M}\left(R_{M}(m) \prec x+m \prec R(x)+\lambda m \prec x\right) .
\end{aligned}
$$

The quintuple $\left(M, \prec, \succ, \beta, R_{M}\right)$ is then called the Hom-associative Rota-Baxter bimodule of weight over the Hom-associative Rota-Baxter algebra A.

Remark 2.1: We have a similar definition for modules over HomLie Rota-Baxter algebras.

The following result is an extension of Lemma 2.5 [29]. It asserts that bimodules over Hom-associative Rota-Baxter algebras are stable under twisting.

Theorem 2.1: Let $\left(M, \prec, \succ, \alpha_{M}, R_{M}\right)$ be a Hom-associative Rota-Baxter bimodule over the Hom-associative Rota-Baxter algebra $(A, \bullet, \alpha, R)$ and be an endomorphism of $A$. Let, $\succ_{\alpha}: A \otimes M \rightarrow M$ and $\prec_{\alpha}: M \otimes A \rightarrow M$ be bilinear maps defined by

$$
{ }_{\beta}=\beta^{\circ}, \quad \succ_{\alpha}=\succ^{\circ}\left(\alpha^{2} \otimes I d_{M}\right) \text { and } \prec_{\alpha}=\prec^{\circ}\left(I d_{M} \otimes \alpha^{2}\right) .
$$

Then $\left(M, \prec_{\alpha}, \succ_{\alpha}, \alpha_{M}, R_{M}\right)$ is a Hom-associative Rota-Baxter 
bimodule of weight $\lambda$ over $\left(A, \cdot{ }_{\beta}, \beta^{\circ} \alpha, R\right)$.

Proof: We prove the compatibility condition (16). For any $x, y \in A$, $m \in M$, one has

$$
\begin{aligned}
\alpha(x) \succ_{\alpha}\left(m \prec_{\alpha} y\right) & =\alpha(x) \succ_{\alpha}\left(m \prec \alpha^{2}(y)\right)=\alpha^{3}(x) \succ\left(m \prec \alpha^{2}(y)\right) \\
& =\left(\alpha^{2}(x) \succ m\right) \prec \alpha^{3}(y)=\left(x \succ_{\alpha} m\right) \prec_{\alpha} \alpha(y) .
\end{aligned}
$$

And

$$
\begin{gathered}
R(x) \succ_{\alpha} R_{M}(m)=R\left(\alpha^{2}(x)\right) \succ R_{M}(m)=R_{M}\left(R\left(\alpha^{2}(x)\right) \succ m+\alpha^{2}(x) \succ R(m)+\lambda \alpha^{2}(x) \succ m\right) \\
=R_{M}\left(R(x) \succ_{\alpha} m+x \succ_{\alpha} R(m)+\lambda x \succ_{\alpha} m\right) .
\end{gathered}
$$

The others relations are proved similarly, and Lemma 1.1 finishes the proof.

We have the below consequences.

Corollary 2.1: Let $\left(M, \prec, \succ, \alpha_{M}, R_{M}\right)$ be a Hom-associative RotaBaxter bimodule of weight $\lambda$ over the multiplicative Hom-associative Rota-Baxter algebra $(A, \bullet, \alpha, R)$. Then $\left(M, \prec, \succ, \alpha_{M}, R_{M}\right)$ is a Homassociative Rota-Baxter bimodule of weight over $\left(A, \alpha^{n \circ}, \alpha^{n+1}, R\right)$ for any entiger $\mathrm{n} \geq 0$.

Corollary 2.2: Let $\left(M, \prec, \succ, \alpha_{M}, R_{M}\right)$ be a Hom-associative RotaBaxter bimodule of weight $\lambda$ over the associative Rota-Baxter algebra $(A, \bullet, \alpha)$ and be an endomorphism of $\mathrm{A}$. Then $\left(M, \prec, \succ, \alpha_{M}, R_{M}\right)$ is a Hom-associative Rota-Baxter bimodule of weight over the multiplicative Hom-associative Rota-Baxter algebra $\left(A, \alpha^{\circ}, \alpha, R\right)$.

Corollary 2.3: Any Hom-associative Rota-Baxter (left) module over a Hom-associative Rota-Baxter algebra $\mathrm{A}$ is a Hom-Lie RotaBaxter (left) module over the Hom-Lie Rota-Baxter algebra associated to $\mathrm{A}$.

Proof: It follows from Lemma 1.2 and Theorem 2.1.

Example 2.1: Any multiplicative Hom-associative Rota-Baxter algebra is a module over itself.

The below theorem shows that Hom-Lie Rota-Baxter modules over Hom-Lie Rota-Baxter algebra are closed under twisting.

Theorem 2.2: Let $\left(M, \succ, \beta, R_{M}\right)$ be a Hom-Lie Rota-Baxter (left) module of weight on the multiplicative Hom-Lie Rota-Baxter algebra $(L,[-,-], \alpha, R)$. Then $\left(M, \succ_{\beta}=\succ^{\circ}\left(\alpha \otimes I d_{M}\right), \beta, R_{M}\right)$ is another Hom-Lie Rota-Baxter (left) module on the Hom-Lie Rota-Baxter algebra L.

Proof: First, for any $x, y \in L, m \in M$ one has

$$
\beta\left(x \succ_{\beta} m\right)=\beta(\alpha(x) \succ m)=\alpha^{2}(x) \succ \beta(m)=\alpha(x) \succ_{\beta}(m) .
$$

Then,

$$
\begin{aligned}
{[x, y] \succ_{\beta} \beta(m) } & =\alpha([x, y]) \succ \beta(m)=[\alpha(x), \alpha(y)] \succ \beta(m) \\
= & \alpha^{2}(x) \succ(\alpha(y) \succ m)-\alpha^{2}(y) \succ\left(\alpha(x) \succ_{m}\right) \\
= & \alpha(x) \succ_{\beta}\left(y \succ_{\beta} m\right)-\alpha(y) \succ_{\beta}\left(x \succ_{\beta} m\right) .
\end{aligned}
$$

Finally,

$$
\begin{aligned}
R(x) \succ_{\beta} R_{M}(m)=\alpha(R(x)) \succ R_{M}(m)=R(\alpha(x)) \succ R_{M}(m) \\
=R_{M}\left(R(\alpha(x)) \succ m+\alpha(x) \succ R_{M}(m)+\lambda \alpha(x) \succ m\right) \\
=R_{M}(\alpha(R(x)) \succ m+\alpha(x) \succ R(m)+\lambda \alpha(x) \succ m) \\
=R_{M}\left(R(x) \succ_{\beta} m+x \succ_{\beta} R_{M}(m)+\lambda x \succ_{\beta} m\right) .
\end{aligned}
$$

This completes the proof.
Corollary 2.4: Let $(A, \bullet, \alpha, R)$ be a multiplicative Hom-associative Rota-Baxter algebra and $\left(M, \succ, \beta, R_{M}\right)$ be a Hom-associative RotaBaxter (left) module over $A_{\alpha}$. Then $\left(M, \succ_{\beta}, \beta, R_{M}\right)$ is a (left)module over the Hom-Lie Rota-Baxter algebra associated to A as in Lemma 2.4.

Corollary 2.5: Let $\left(M, \succ_{\beta}, \beta, R_{M}\right)$ be a Hom-Lie (left) module of weight $\lambda$ over the multiplicative Hom-Lie Rota-Baxter algebra $L_{\alpha}=\left(L,[-,-]_{\alpha}=\alpha^{\circ}[-,-], \alpha\right)$. Then $\left(M, \succ_{\beta}, \beta, R_{M}\right)$ is another HomLie Rota-Baxter (left) module over $L_{\alpha}$.

The rest of this section is dedicated to bimodules over Hom-preLie algebra from Hom-associative Rota-Baxter bimodules.

Definition 2.2: A non-associative algebra $S$ with the linear map $\alpha$ : $S \rightarrow S$ and the multiplication $(x, y) \mapsto x \cdot y$ is called Hom-preLie algebra if the following Hom-preLie identity is satisfied [29]

$(x \cdot y) \cdot \alpha(z)-\alpha(x) \cdot(y \cdot z)=(y \cdot x) \cdot \alpha(z)-\alpha(y) \cdot(x \cdot z)$,

for all $x, y, z \in S$.

Definition 2.3: Let $\left(S,{ }^{\circ}, \alpha\right)$ beaHom-preLiealgebra.AnS-bimodule is a vector space $M$ endowed with a linear map $\beta: M \rightarrow M$, two bilinear maps $\succ: S \otimes M \rightarrow M, x \otimes m \mapsto x \succ m \quad$ and $\quad \prec: M \otimes S \rightarrow M, m \otimes x \mapsto m \prec x$ ,such that $\beta(x \succ m)=\alpha(x) \succ \beta(m), \beta(m \prec x)=\beta(m) \prec \alpha(x)$

and

$\alpha(x) \succ(y \succ m)-(x \cdot y) \succ \beta(m)-\alpha(y) \succ(x \succ m)+(y \cdot x) \succ \beta(m)=0$,

$\alpha(x) \succ(m \prec y)-(x \succ m) \prec \alpha(y)-\beta(m) \prec(x \cdot y)+(m \prec x) \prec \alpha(y)=0$.

Lemma 2.1: Let $\left(A,{ }^{*}, \alpha\right)$ be a Hom-associative Rota-Baxter algebra weight 0 . We define the operation on $\mathrm{A}$ by [22]

$x * y=R(x) \cdot y-y \cdot R(x)$.

Then $\left(A,{ }^{*}, \alpha\right)$ is a Hom-preLie algebra.

Theorem 2.3: Let $\left(M, \prec, \succ, \beta, R_{M}\right)$ be a Hom-associative RotaBaxterbimoduleover theHom-associative Rota-Baxteralgebra $(A, \bullet, \alpha, R)$. Define two bilinear maps $\quad A \otimes M \rightarrow M$ and $\triangleleft: M \otimes A \rightarrow M$ by $x \triangleright m=R(x) \succ m-m \prec R(x)$ and $m \triangleleft x=R(x) \succ m-m \prec R(x)$.

Then $(M, \triangleleft, \triangleright, \beta)$ is a bimodule over the Hom-preLie algebra $\left(A,{ }^{*}, \alpha\right)$ associated to $\mathrm{A}$.

Proof: For any $x, y \in A, m \in M$, one has

$$
\begin{aligned}
\alpha(x) \triangleright(y \triangleright m)= & \alpha(x) \triangleright(R(x) \succ m-m \prec R(x) \\
= & R(\alpha(x)) \succ(R(y) \succ m-m \prec R(y))-(R(y) \succ m-m \prec R(y)) \prec R(\alpha(x)) \\
= & R(\alpha(x)) \succ(R(y) \succ m)-R(\alpha(x)) \succ(m \prec R(y)) \\
& -(R(y) \succ m) \prec R(\alpha(x))+(m \prec R(y)) \prec R(\alpha(x)) \\
= & \alpha(R(x)) \succ(R(y) \succ m)-\alpha(R(x)) \succ(m \prec R(y)) \\
& -(R(y) \succ m) \prec \alpha(R(x))+(m \prec R(y)) \prec \alpha(R(x)) . \\
(x * y) \triangleright \beta(m) & =(R(x) \cdot y-y \cdot R(x)) \triangleright \beta(m) \\
& =R(R(x) \cdot y-y \cdot R(x)) \succ \beta(m)-\beta(m) \prec R(R(x) \cdot y-y \cdot R(x) \\
& =R(R(x) \cdot y) \succ \beta(m)-R(y \cdot R(x)) \succ \beta(m) \\
& -\beta(m) \prec R(R(x) \cdot y)+\beta(m) \prec R(y \cdot R(x)) .
\end{aligned}
$$

Then,

$\alpha(x) \triangleright(y \triangleright m)-(x * y) \triangleright \beta(m)-\alpha(y) \triangleright(x \triangleright m)+(y * x) \triangleright \beta(m)=$ $=\alpha(R(x)) \succ(R(y) \succ m)-\alpha(R(x)) \succ(m \prec R(y))-(R(y) \succ m) \prec \alpha(R(x))+(m \prec R(y)) \prec \alpha(R(x))$ $-R(R(x) \cdot y) \succ \beta(m)+R(y \cdot R(x)) \succ \beta(m)+\beta(m) \prec R(R(x) \cdot y)-\beta(m) \prec R(y \cdot R(x))$ $-\alpha(R(y)) \succ(R(x) \succ m)+\alpha(R(y)) \succ(m \prec R(x))+(R(x) \succ m) \prec \alpha(R(y))+(m \prec R(x)) \prec \alpha(R(y))$ $+R(R(y) \cdot x) \succ \beta(m)+R(x \cdot R(y)) \succ \beta(m)+\beta(m) \prec R(R(y) \cdot x)-\beta(m) \prec R(x \cdot R(y))$ 
$\alpha(x) \triangleright(y \triangleright m)-(x * y) \triangleright \beta(m)-\alpha(y) \triangleright(x \triangleright m)+(y * x) \triangleright \beta(m)=$ $\alpha(R(x)) \succ(R(y) \succ m)-\alpha(R(x)) \succ(m \prec R(y))-(R(y) \succ m) \prec \alpha(R(x))+(m \prec R(y)) \prec \alpha(R(x))$ $-(R(x) \cdot R(y)) \succ \beta(m)+(R(y) \cdot R(x)) \succ \beta(m)+\beta(m) \prec(R(x) \cdot R(y))-\beta(m) \prec(R(y) \cdot R(x))$ $-\alpha(R(y)) \succ(R(x) \succ m)+\alpha(R(y)) \succ(m \prec R(x))+(R(x) \succ m) \prec \alpha(R(y))+(m \prec R(x)) \prec \alpha(R(y))$

The second relation is proved analogously.

Proposition 2.1: Let $(L,[-,-], \alpha, R)$ be a Hom-Lie Rota-Baxter algebra (of weight 0$)$ and $(M, \succ, \beta)$ be the sub-adjacent Hom-Lie module. Let $\triangleright: L \otimes M \rightarrow M$ be a bilinear map defined by $x \triangleright m=R(x) \succ m$.

Then $(M, \triangleright, \beta)$ is a (left) module over the Hom-preLie algebra $\left(L,[-,-]^{\circ}(R \otimes I d), \alpha\right)$.

Proof: For any $x, y \in A, m \in M$, one has

$\alpha(x) \triangleright(y \triangleright m)-(x \cdot y) \triangleright \beta(m)-\alpha(y) \triangleright(x \triangleright m)+(y \cdot x) \triangleright \beta(m)=$

$=\alpha(x) \triangleright(R(y) \succ m)-[R(x), y] \succ \beta(m)-\alpha(y) \triangleright(R(x) \succ m)+[R(y), x] \triangleright \beta(m)$

$=R(\alpha(x)) \succ(R(y) \succ m)-R([R(x), y]) \succ \beta(m)-R(\alpha(y)) \succ(R(x) \succ m)+R([R(y), x]) \succ \beta(m)$

$=\alpha(R(x)) \succ(R(y) \succ m)-\alpha(R(y)) \succ(R(x) \succ m)-R([R(x), y])+R([x, R(y)]) \succ \beta(m)$

$=[R(x), R(y)] \succ \beta(m)-[R(x), R(y)] \succ \beta(m)=0$.

This finishes the proof.

Lemma 2.2: Let $(A, \bullet, \alpha, R)$ be a Hom-associative Rota-Baxter algebra of weight 1 . We define the operation on A by [30]

$x * y=R(x) \cdot y-y \cdot R(x)-x \cdot y$

Then $(A, *, \alpha)$ is a Hom-preLie algebra.

Theorem 2.4: Let $\left(M, \prec, \succ, \beta, R_{M}\right)$ be a Hom-associative RotaBaxter bimodule of weight 1 over the Hom-associative Rota-Baxter algebra $(A, \bullet, \alpha, R)$ of weight 1 . Let us define bilinear maps .

$$
\begin{aligned}
& x \triangleright m=R(x) \succ m-m \prec R(x)-x \succ m \\
& m \triangleleft x=R_{M}(m) \prec x-x \succ R_{M}(m)-m \prec x
\end{aligned}
$$

Then $(M, \triangleleft, \triangleright, \beta)$ is a bimodule over the Hom-preLie algebra of Lemma 2.2.

Proof: The proof is similar to the one of Theorem 2.3.

\section{Q-Hom-tridendriform Algebras}

In this section, we introduce Rota-Baxter q-Hom-tridendriform algebras. They espouse the most properties of Hom-tridendriform algebras [31], so we omit them. Then we express axioms difining q-Hom-tridendriform algebras by mean of vector basis.

Definition 3.1: Let $\mathrm{q} \in \boldsymbol{K}$. A q-Hom-tridendriform algebra is a quintuple $(D, \dashv, \vdash, \cdot, \alpha)$ consisting of a vector space $\mathrm{D}$ on which the operations $\dashv, \vdash, \cdot D \otimes D \rightarrow D$ are bilinear maps and $\alpha: D \rightarrow D$ is a linear map satisfing:

$$
\begin{aligned}
& x \dashv y) \dashv \alpha(z)=\alpha(x) \dashv(y \dashv z+y \vdash z+q y \cdot z), \\
& (x \vdash y) \dashv \alpha(z)=\alpha(x) \vdash(y \dashv z), \\
& \alpha(x) \vdash(y \vdash z)=(x \dashv y+x \vdash y+q x \cdot y) \vdash \alpha(z), \\
& (x \dashv y) \cdot \alpha(z)=\alpha(x) \cdot(y \vdash z), \\
& (x \vdash y) \cdot \alpha(z)=\alpha(x) \vdash(y \cdot z), \\
& (x \cdot y) \dashv \alpha(z)=\alpha(x) \cdot(y \dashv z), \\
& (x \cdot y) \cdot \alpha(z)=\alpha(x) \cdot(y \cdot z),
\end{aligned}
$$

for $x, y, z \in D$.
It is clear that for any $q \neq 0,\left(D, \dashv, \vdash, q^{-1}, \alpha\right)$ turn to Homtridendriform algebra when-ever $(D, \dashv, \vdash,, \alpha)$ is a q-Homtridendriform algebra.

Example 3.1: i) Any q-tridendriform algebra is a q-Homtridendriform algebra with $\alpha=I d$.

ii) A Hom-tridendriform algebra is q-Hom-tridendriform algebra with $\mathrm{q}=1$.

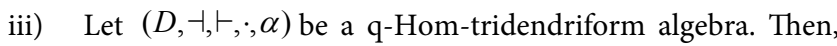
for any parameters $\lambda, \theta \in \mathbb{K}$,

$$
\left(D, \dashv \lambda:=\lambda \dashv, \vdash \lambda:=\lambda \vdash, \cdot \lambda:=\lambda \cdot, \alpha_{\theta}:=\theta \alpha\right)
$$

is also a q-Hom-tridendriform algebra.

Definition 3.2: A q-Hom-tridendriform algebra $(D, \dashv, \vdash,, \alpha)$ endowed with a linear map $R: D \rightarrow D$ satisfing

$$
\begin{aligned}
& R^{\circ} \alpha=\alpha^{\circ} R \\
& R(x) \dashv R(y)=R(x \dashv R(y)+R(x) \dashv y+\lambda x \dashv y), \\
& R(x) \vdash R(y)=R(x \vdash R(y)+R(x) \vdash y+\lambda x \vdash y), \\
& R(x) \cdot R(y)=R(x \cdot R(y)+R(x) \cdot y+\lambda x \cdot y) .
\end{aligned}
$$
$\mathbb{K}$.

is called q-Hom-tridendriform Rota-Baxter algebra of weight $\lambda \in$

Remark 3.1: We have a similar definition for Hom-Poisson RotaBaxter algebra (with two binary operations) which is a Hom-Poisson algebra endowed with a linear operator that commutes with the twisting map and satisfies the Rota-Baxter relations for both associative and Hom-Lie product. By a direct computation we can show that every Hom-associative Rota-Baxter algebra has a non-commutative HomPoisson Rota-Baxter algebra structure in which the Hom-Poisson bracket is the commutator bracket. It is also easy to prove that RotaBaxter Poisson algebras turn to Hom-Poisson Rota-Baxter algebras by twisting the Poisson Rota-Baxter algebra structure. By a straightforward calculation, we may prove that any Hom-Lie Rota-Baxter algebra is a Hom-Lie admissible Rota-Baxter algebra with the same twisting map and Rota-Baxter operator.

Definition 3.3: Let $(D, \dashv, \vdash,, \alpha)$ be a q-Hom-tridendriform algebra. A morphism of q-Hom-tridendriform algebra is a linear map $\beta: D \rightarrow D$ such that

$\beta^{\circ} \alpha=\alpha^{\circ} \beta, \quad \beta(x \dashv y)=\beta(x) \dashv \beta(y), \quad \beta(x \vdash x)=\beta(y) \vdash \beta(x), \quad \beta(x \cdot y)=\beta(x) \cdot \beta(y)$, for any $x, y D$.

Definition 3.4: A q-Hom-tridendriform $(D, \dashv, \vdash, \cdot, \alpha)$ in which is a morphism is said to be a multiplicative q-Hom-tridendriform algebra.

Theorem 3.1: Let $(A, \bullet, \alpha, R)$ be a Hom-associative Rota-Baxter algebra of weight q. Then $(A, \dashv, \vdash,, \alpha)$ is a q-Hom-tridendriform RotaBaxter algebra (of weight q), where $x \dashv y=x \cdot R(y), x \vdash y=R(x) \cdot y$.

Proof: It is proved by a direct computation.

In what follows, we express by mean of vector basis the conditions difining a finite dimensional q-Hom-tridendriform algebra $(D, \dashv, \vdash,, \alpha)$ and the Rota-Baxter operator on it. Let $\left\{e_{j}\right\}, i=1, \ldots, N$ be basis of $\mathrm{D}$. For any $i, j \in \mathbb{N}, 1 \leq I, j \leq N$, let us put

$$
e_{i} \dashv e_{j}=a_{i j}^{k} e_{k}, \quad e_{i} \vdash e_{j}=b_{i j}^{k} e_{k}, \quad e_{i} \cdot e_{j}=c_{i j}^{k} e_{k} \quad \text { and } \quad \alpha\left(e_{i}\right)=\alpha_{i}^{j} e_{j} .
$$

Then we have the below conclusions. 
Lemma 3.1: The axioms in Definition 3.1 are respectively equivalent to

$$
\begin{aligned}
& a_{i j}^{l} \alpha_{k}^{m} a_{l m}^{n}=\alpha_{i}^{k} a_{j k}^{m} a_{l m}^{n}+\alpha_{i}^{l} b_{j k}^{m} a_{l m}^{n}+q \alpha_{i}^{l} c_{j k}^{m} a_{l m}^{n}, \\
& b_{i j}^{l} \alpha_{k}^{m} a_{l m}^{n}=\alpha_{i}^{m} a_{j k}^{l} b_{m l}^{n}, \\
& \alpha_{i}^{l} b_{j k}^{m} b_{l m}^{n}=a_{i j}^{l} \alpha_{k}^{n} b_{l m}^{n}+b_{i j}^{l} \alpha_{k}^{m} b_{l m}^{n}+q c_{i j}^{l} \alpha_{k}^{m} b_{l m}^{n}, \\
& a_{i j}^{l} \alpha_{k}^{m} c_{l m}^{n}=\alpha_{i}^{l} b_{j k}^{m} c_{l m}^{n}, \\
& b_{i j}^{l} \alpha_{k}^{m} c_{l m}^{n}=\alpha_{i}^{l} c_{j k}^{m} b_{l m}^{n}, \\
& c_{i l} \alpha_{k}^{m} a_{l m}^{n}=\alpha_{i}^{l} a_{j k}^{m} c_{l m}^{n}, \\
& c_{i j}^{l} \alpha_{k}^{m} c_{l m}^{n}=\alpha_{i}^{l} c_{j k}^{m} c_{l m}^{n},
\end{aligned}
$$

for any $i, j, k, l, m, n \in \mathbb{N}, 1 \leq i, j, k, l, m, n \leq N$.

Lemma 3.2: Let $R$ be a Rota-Baxter operator of weight $\lambda$ on a $\mathrm{q}-\mathrm{Hom}$-tridendriform algebra of dimension $\mathrm{N}$. Then in terms of basis elements, relations (31)-(34) are respectively equivalent to the following

$$
\begin{aligned}
& r_{i}^{j} \alpha_{j}^{k}=\alpha_{i}^{j} r_{j}^{k}, \\
& r_{i}^{k} r_{j}^{l} a_{k l}^{m}=r_{i}^{k} a_{k j}^{l} r_{l}^{m}+r_{j}^{k} a_{i k}^{l} r_{l}^{m}+\lambda a_{i j}^{k} r_{k}^{m}, \\
& r_{i}^{k} r_{j}^{l} b_{k l}^{m}=r_{i}^{k} b_{k j}^{l} r_{l}^{m}+r_{j}^{k} b_{i k}^{l} r_{l}^{m}+\lambda b_{i j}^{k} r_{k}^{m}, \\
& r_{i}^{k} r_{j}^{l} c_{k l}^{m}=r_{i}^{k} c_{k j}^{l} r_{l}^{m}+r_{j}^{k} c_{i k}^{l} r_{l}^{m}+\lambda c_{i j}^{k} r_{k}^{m},
\end{aligned}
$$

for any $i, j, k, l, m, n \in \mathbb{N}, 1 \leq i, j, k, l, m, n \leq N$.

Theorem 3.2: The linear map $\mathrm{R}$ is a Rota-Baxter operator of weight $\lambda$ on an $\mathrm{N}$-dimensional q-Hom-tridendriform algebra if, and only if, relations (35)-(45) hold.

\section{q-Hom-tridendriform Bimodules}

In this section, we introduce bimodules over q-Hom-tridendriform algebras. We give some examples and prove that they are closed by twisting. Then we give their connection with Hom-associative RotaBaxter bimodules.

Definition 4.1: Let $(D, \dashv, \vdash,, \alpha)$ be a q-Hom-tridendriform algebra. A D-bimodule is a vector space $\mathrm{M}$ together with a linear map $\beta: M \rightarrow M$ and six bilinear maps

$$
\begin{array}{lcc}
D \otimes M \rightarrow M & D \otimes M \rightarrow M & D \otimes M \rightarrow M \\
x \otimes m \mapsto x \triangleright m & x \otimes m \mapsto x \triangleleft m & x \otimes m \mapsto x \succ m \\
M \otimes D \rightarrow M & M \otimes D \rightarrow M & M \otimes D \rightarrow M \\
m \otimes x \mapsto m \triangleright x & m \otimes x \mapsto m \triangleleft x & m \otimes x \mapsto m \prec x
\end{array}
$$

such that

$\beta(x \triangleleft m)=\alpha(x) \triangleleft \beta(m), \quad \beta(m \triangleleft x)=\beta(m) \triangleleft \alpha(x), \quad \beta(x \triangleright m)=\alpha(x) \triangleright \beta(m)$,

$\beta(m \triangleright x)=\beta(m) \triangleright \alpha(x), \quad \beta(x \succ m)=\alpha(x) \succ \beta(m), \quad \beta(m \prec x)=\beta(m) \prec \alpha(x)$, and

$$
(x \dashv y) \triangleleft \beta(m)=\alpha(x) \triangleleft(y \triangleleft m+y \triangleright m+q y \succ m),
$$

$$
\begin{aligned}
& (x \vdash y) \triangleleft \beta(m)=\alpha(x) \triangleright(y \triangleleft m), \\
& \alpha(x) \triangleright(y \triangleright m)=(x \dashv y+x \vdash y+q x \cdot y) \triangleright \beta(m), \\
& (x \dashv y) \succ \beta(m)=\alpha(x) \succ(y \triangleright m), \\
& (x \vdash y) \succ \beta(m)=\alpha(x) \triangleright(y \succ m), \\
& (x \cdot y) \triangleleft \beta(m)=\alpha(x) \succ(y \triangleleft m), \\
& (x \cdot y) \succ \beta(m)=\alpha(x) \succ(y \succ m) . \\
& (x \triangleleft m) \triangleleft \alpha(y)=\alpha(x) \triangleleft(m \triangleleft y+m \triangleright y+q m \prec y), \\
& (x \triangleright m) \triangleleft \alpha(y)=\alpha(x) \triangleright(m \triangleleft y), \\
& \alpha(x) \triangleright(m \triangleright y)=(x \triangleleft m+x \triangleright m+q x \succ m) \triangleright \alpha(y), \\
& (x \triangleleft m) \prec \alpha(y)=\alpha(x) \succ(m \triangleright y), \\
& (x \triangleright m) \prec \alpha(y)=\alpha(x) \triangleright(m \prec y), \\
& (x \succ m) \triangleleft \alpha(y)=\alpha(x) \succ(m \triangleleft y), \\
& (x \succ m) \prec \alpha(y)=\alpha(x) \succ(m \prec y) . \\
& (m \triangleleft x) \triangleleft \alpha(y)=\beta(m) \triangleleft(x \dashv y+x \vdash y+q x \cdot y), \\
& (m \triangleright x) \triangleleft \alpha(y)=\beta(m) \triangleright(x \dashv y), \\
& \beta(m) \triangleright(x \vdash y)=(m \triangleleft x+m \triangleright x+q m \prec x) \triangleright \alpha(y), \\
& (m \triangleleft x) \prec \alpha(y)=\beta(m) \prec(x \vdash y), \\
& (m \triangleright x) \prec \alpha(y)=\beta(m) \triangleright(x \cdot y), \\
& (m \prec x) \triangleleft \alpha(y)=\beta(m) \prec(x \dashv y), \\
& (m \prec x) \prec \alpha(y)=\beta(m) \prec(x \cdot y) .
\end{aligned}
$$

Remark 4.1: 1 . When $\alpha=I d_{D}$, we get the definition of bimodule over q-tridendriform algebra.

2. When $=\prec=\succ=0$, we recover bimodule over Homdendriform algebra [10].

3. When $\cdot=\prec=\succ=0$ and $\alpha=I d_{D}$, we recover bimodule over dendriform algebra [2].

Example 4.1: a) Any q-Hom-tridendriform algebra is a bimodule over itself.

b) Let $\left(M_{1}, \triangleleft_{1}, \triangleright_{1}, \beta_{1}\right)$ and $\left(M_{2}, \triangleleft_{2}, \triangleright_{2}, \beta_{2}\right)$ be two bimodules over a q-Hom-tridendriform algebra $(D, \dashv, \vdash,, \alpha)$. Then the direct product $M=$ $M_{1} \times M_{2}$ is a bimodule over the q-Hom-tridendriform algebra $\mathrm{D}$ with structuremaps $\triangleleft: M \otimes A \rightarrow M, \triangleright: A \otimes M \rightarrow M$ and $\beta: M \rightarrow M$ definedby $\triangleleft\left(x,\left(m_{1}, m_{2}\right)\right)=\left(\triangleleft_{1}\left(x, m_{1}\right), \triangleleft_{2}\left(x, m_{2}\right)\right), \triangleright\left(x,\left(m_{1}, m_{2}\right)\right)=\left(\triangleright_{1}\left(x, m_{1}\right), \triangleright_{2}\left(x, m_{2}\right)\right)$ and $\beta\left(m_{1}, m_{2}\right)=\left(\beta_{1}\left(m_{1}\right), \beta_{2}\left(m_{2}\right)\right)$.

The next result show that bimodules over a q-Hom-tridendriform algebra give rise to twisted bimodules over the corresponding twisted q-Hom-tridendriform algebra.

Theorem 4.1: Let $(M, \triangleleft, \triangleright, \prec, \succ)$ be a bimodule over aqtridendriform algebra $(D, \dashv, \vdash, \cdot)$. Let $\alpha: D \rightarrow D$ be a q-tridendriform algebra endomorphism and $\beta: M \rightarrow M$ be a linear map such that 
$\beta(x \triangleleft m)=\alpha(x) \triangleleft \beta(m), \quad \beta(m \triangleleft x)=\beta(m) \triangleleft \alpha(x), \quad \beta(x \triangleright m)=\alpha(x) \triangleright \beta(m)$, $\beta(m \triangleright x)=\beta(m) \triangleright \alpha(x), \quad \beta(x \succ m)=\alpha(x) \succ \beta(m), \quad \beta(m \prec x)=\beta(m) \prec \alpha(x)$

\section{Define}

$$
\begin{array}{lcrl}
\triangleright_{\beta}: D \otimes M \rightarrow M & \triangleleft_{\beta}: D \otimes M \rightarrow M & \succ_{\beta}: D \otimes M \rightarrow M \\
x \otimes m \mapsto \alpha(x) \triangleright \beta(m), & x \otimes m \mapsto \alpha(x) \triangleleft \beta(m), & x \otimes m \mapsto \alpha(x) \succ \beta(m) \\
\triangleright_{\beta}: M \otimes D \rightarrow M & \triangleleft_{\beta}: M \otimes D \rightarrow M & \prec{ }_{\beta} M \otimes D \rightarrow M \\
m \otimes x \mapsto \beta(m) \triangleright \alpha(x), & m \otimes x \mapsto \beta(m) \triangleleft \alpha(x), & m \otimes x \mapsto \beta(m) \prec \alpha(x) .
\end{array}
$$

Then, $\left(M, \triangleleft_{\beta}, \triangleright_{\beta}, \prec_{\beta}, \succ_{\beta}, \beta\right)$ is a bimodule over the multiplicative q-Hom-tridendriform alge-bra $D_{\alpha}=\left(D, \prec_{\alpha}, \succ_{\alpha},{ }_{\alpha}, \alpha\right)$, where $\prec_{\alpha}=\alpha^{\circ} \prec, \succ_{\alpha}=\alpha^{\circ} \succ$ and $\cdot \alpha=\alpha^{\circ}$.

Proof: The proof is based on the following two remarks:

First, observe that taking ${ }^{\circ}=-1, \vdash$ or $\bullet$ and $\odot=\triangleleft, \prec$ or , $\succ$ the terms occuring in Definition 4.1 may be written

$\left(x^{\circ} y\right) \odot \beta(m), \quad \alpha(x) \odot(y \odot m), \quad(x \odot m) \odot \alpha(y)$,

$\alpha(x) \odot(m \odot y), \quad(m \odot x) \odot \alpha(y), \quad \beta(m) \odot\left(x^{\circ} y\right)$,

for any $x, y \in D, m \in M$.

Next,

$\left(x_{\alpha}^{\circ} y\right) \odot_{\beta} \beta(m)=\beta\left[\alpha\left(x^{\circ} y\right) \odot \beta(m)\right]=\beta^{2}\left[\left(x^{\circ} y\right) \odot m\right]$,

$\alpha(x) \odot_{\beta}(y \odot \beta)=\beta[\alpha(x) \odot \beta(y \odot m)]=\beta^{2}[x \odot(y \odot m)]$,

$\left(x \odot_{\beta} m\right) \odot_{\beta} \alpha(y)=\beta[\beta(x \odot m) \odot \alpha(y)]=\beta^{2}[(x \odot m) \odot y]$,

$\alpha(x) \odot_{\beta}\left(m \odot_{\beta} y\right)=\beta\left[\alpha(x) \odot \beta(m \odot y)=\beta^{2}[x \odot(m \odot y)]\right.$,

$\left(m \odot_{\beta} x\right) \odot_{\beta} \alpha(y)=\beta[\beta(m \odot x) \odot \alpha(y)]=\beta^{2}[(m \odot x) \odot y]$,

$\beta(m) \odot_{\beta}\left(x_{\alpha}^{\circ} y\right)=\beta\left[\beta(m) \odot \alpha\left(x^{\circ} y\right)=\beta^{2}\left[m \odot\left(x^{\circ} y\right)\right]\right.$.

Thus, the proof finishes by using identities (46)-(66) whenever $\alpha=I d_{D}$ and $\beta=I d_{M}$.

Now we have the following series of lemmas.

It is known [25] that if $(A, \bullet, \alpha, R)$ is a Hom-associative Rota-Baxter algebra. Then, $\left(A,{ }^{*}, \alpha, R\right)$ is a Hom-associative Rota-Baxter algebra, where $x * y=R(x) \cdot y+x \cdot R(y)+\lambda x \cdot y$. Then we have the following proposition.

Lemma 4.1: Let $\left(M, \prec, \succ, \beta, R_{M}\right)$ be a Hom-associative Rota-Baxter bimodule of weight $\lambda$ over the Hom-associative Rota-Baxter algebra $(A, \bullet, \alpha, R)$. Define bilinear maps $\triangleleft: M \otimes A \rightarrow M$ and $\triangleright: A \otimes M \rightarrow M$ by

$x \triangleright m=R(x) \succ m+x \succ R_{M}(m)+\lambda x \succ m, m \triangleleft x=R_{M}(x) \prec x+m \prec R(x)+\lambda m \prec x$.

Then $\left(M, \triangleleft, \triangleright, \beta, R_{M}\right)$ is a Hom-associative Rota-Baxter bimodule of weight $\lambda$ over the Hom-associative algebra $\left(A,{ }^{*}, \alpha\right)$.

Proof: First, for any $x \in A, m \in M$,

$$
\begin{aligned}
R(x) \triangleright R_{M}(m) & =R^{2}(x) \succ R(m)+R(x) \succ R_{M}^{2}(m)+\lambda R(x) \succ R(m) \\
& =R_{M}\left(R^{2}(x) \succ m+R(x) \succ R_{M}(m)+\lambda R(x) \succ m\right. \\
& +R(x) \succ R(m)+x \succ R_{M}^{2}(m)+\lambda x \succ R(m) \\
& \left.+R(x) \succ m+x \succ R_{M}(m)+\lambda x \succ m\right) \\
& =R_{M}\left(R(x) \triangleright m+x \triangleright R_{M}(m)+\lambda x \triangleright m\right) .
\end{aligned}
$$

Next, for any $x, y \in A, m \in M$, one has

$$
\begin{aligned}
(x * y) \triangleright \beta(m)= & (R(x) \cdot y+x \cdot R(y)+\lambda x \cdot y) \triangleright \beta(m) \\
= & R(R(x) \cdot y+x \cdot R(y)+\lambda x \cdot y) \succ \beta(m) \\
& +(R(x) \cdot y+x \cdot R(y)+\lambda x \cdot y) \succ R_{M}(\beta(m)) \\
& +\lambda(R(x) \cdot y+x \cdot R(y)+\lambda x \cdot y) \succ \beta(m) \\
= & (R(x) \cdot R(y)) \succ \beta(m)+(R(x) \cdot y) \succ R_{M}(\beta(m)) \\
& +(x \cdot R(y)) \succ R_{M}(\beta(m))+\lambda(x \cdot y) \succ R_{M}(\beta(m)) \\
& +\lambda(R(x) \cdot y) \succ \beta(m)+\lambda(x \cdot R(y)) \succ \beta(m)+\lambda^{2}(x \cdot y) \succ \beta(m) .
\end{aligned}
$$

By axiom (14),

$$
\begin{aligned}
(x * y) \triangleright \beta(m)= & R(\alpha(x)) \succ\left(R(y) \succ m+y \succ R_{M}(m)+\lambda y \succ m\right)-\lambda R(\alpha(x)) \succ(y \succ m) \\
+ & \alpha(x) \succ R_{M}(y \triangleright m)+\lambda \alpha(x) \succ\left(y \succ R_{M}(m)\right) \\
+ & \lambda(R(x) \cdot y) \succ \beta(m)+\lambda(x \cdot R(y)) \succ \beta(m)+\lambda^{2}(x \cdot y) \succ \beta(m) \\
= & R(\alpha(x)) \succ(y \triangleright m)+\alpha(x) \succ R_{M}(y \triangleright m) \\
& +\lambda \alpha(x) \succ\left(R(y) \succ m+y \succ R_{M}(m)+\lambda y \succ m\right) \\
= & R(\alpha(x)) \succ(y \triangleright m)+\alpha(x) \succ R_{M}(y \triangleright m)+\lambda \alpha(x) \succ(y \triangleright m) \\
= & \alpha(x) \triangleright(y \triangleright m) .
\end{aligned}
$$

The others relations are proved similarly.

Corollary 4.1: Let $\left(M, \succ, \beta, R_{M}\right)$ be a Hom-associative RotaBaxter (left) module of weight $\lambda$ over the Hom-associative Rota-Baxter algebra $(A, \cdot, \alpha, R)$. Then $\left(M, \triangleright, \beta, R_{M}\right)$ is also a Hom-Lie Rota-Baxter (left)module of weight $\lambda$ over the Hom-Lie Rota-Baxter algebra of Lemma 1.2.

Corollary 4.2: Let $\left(M, \succ, \beta, R_{M}\right)$ be a Hom-associative RotaBaxter (left) module of weight $\lambda$ over the Hom-associative Rota-Baxter algebra $(A, \cdot, \alpha, R)$. Then $\left(M, \triangleright_{\alpha}=\triangleright^{\circ}\left(\alpha^{2} \otimes I d_{M}\right), \beta, R_{M}\right)$ is also a Homassociative Rota-Baxter (left) module over $(A,, \alpha, R)$.

Definition 4.2: A q-Hom-tridendriform Rota-Baxter bimodule $\left(M, \triangleleft, \triangleright, \prec, \succ, R_{M}, \beta\right)$ is a Hom-associative Rota-Baxter bimodules $\left(M, \prec, \succ, R_{M}, \beta\right)$ satisfying

$$
\begin{aligned}
& R(x) \triangleleft R_{M}(m)=R_{M}\left(R(x) \triangleleft m+x \triangleleft R_{M}(m)+\lambda x \triangleleft m\right), \\
& R_{M}(m) \triangleleft R(x)=R_{M}\left(R_{M}(m) \triangleleft x+m \triangleleft R(x)+\lambda m \triangleleft x\right), \\
& R(x) \triangleright R_{M}(m)=R_{M}\left(R(x) \triangleright m+x \triangleright R_{M}(m)+\lambda x \triangleright m\right), \\
& R_{M}(m) \triangleright R(x)=R_{M}\left(R_{M}(m) \triangleright x+m \triangleright R(x)+\lambda m \triangleright x\right) .
\end{aligned}
$$

Below lemmas and corollaries are proved similarly to the one of Lemma 4.1.

Lemma 4.2: Let $\left(M, \prec, \succ, \beta, R_{M}\right)$ be a Hom-associative RotaBaxter bimodule of weight $\lambda$ over the Hom-associative Rota-Baxter algebra $(A, \bullet, \alpha, R)$ (of weight $\lambda$ ). Define bilinear maps $\triangleleft: M \otimes A \rightarrow M$, and $\triangleright: A \otimes M \rightarrow M, \succ^{\prime}: A \otimes M \rightarrow M$ and $\prec^{\prime}: M \otimes A \rightarrow M$ by

$$
\begin{aligned}
& x \triangleleft m=x \succ R_{M}(m), \quad x \triangleright m=R(x) \succ m, \quad x \succ^{\prime} m=x \succ m, \\
& m \triangleleft x=m \prec R(x), \quad m \triangleright x=R_{M}(m) \prec x, \quad m \prec x=m \prec x .
\end{aligned}
$$

Then $\left(M, \triangleleft, \triangleright, \prec^{\prime}, \succ^{\prime}, R_{M}, \beta\right)$ is a $\mathrm{q}$-Hom-tridendriform RotaBaxter bimodule (of weight ) over the q-Hom-tridendriform algebra $(A, \dashv, \vdash,, \alpha)$, where, $x \dashv y:=x \cdot R(y), x \vdash y:=R(x) \cdot y$.

Lemma 4.3: Let $(M, \triangleleft, \triangleright, \prec, \succ, \beta)$ be a q-Hom-tridendriform bimodule over the q-Hom-tridendriform $(A, \dashv, \vdash, \cdot, \alpha)$. Define bilinear maps $\triangleleft: M \otimes A \rightarrow M$ and $\triangleright: A \otimes M \rightarrow M$ by 
$x \triangleright^{\prime} m=x \triangleright m+q x \succ m, \quad x \triangleleft^{\prime} m=x \triangleleft m$,

$m \triangleright^{\prime} x=m \triangleright x+q m \prec x, \quad m \triangleleft^{\prime} x=m \triangleleft x$.

Then $\left(M, \triangleleft^{\prime}, \triangleright^{\prime}, \beta\right)$ is a Hom-dendriform bimodule over the Homdendriform algebra $(A, \dashv, \vdash+q \cdot, \alpha)$.

Lemma 4.4: [10] Let $(M, \prec, \succ, \beta)$ be a bimodule over the Homdendriform $(D, \dashv, \vdash, \alpha)$. Define bilinear maps $\triangleleft: M \otimes A \rightarrow M$ and $\triangleright: A \otimes M \rightarrow M$ by

$x \triangleleft m=x \succ m+x \prec m, \quad m \triangleright x=m \succ x+m \prec x$

Then $(M, \triangleleft, \triangleright, \beta)$ is a bimodule over the Hom-associative algebra $(A, \dashv+\vdash, \alpha)$.

Let us Denote by HARBM: the category of Hom-associative RotaBaxter bimodules, q-HTDM : the category of q-Hom-tridendriform bimodules, HDM : the category of Hom-dendriform bimodules and HA : the category of Hom-associative bimodules. Then, the above discussion may be summarized in the following theorem.

Theorem 4.2: The following diagram is commutative

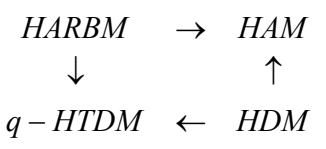

Proof: The top horizontal arrow and the bottom horizontal arrow follow from Lemma 4.1 and Lemma 4.4 respectively. The left vertical arrow is established in Lemma 4.2 and the right vertical arrow is the functor constructed in Lemma 4.3.

Corollary 4.3: Let $\left(M, \prec, \succ, \beta, R_{M}\right)$ be a Rota-Baxter bimodule of weight over the Hom-associative Rota-Baxter algebra $(A, \bullet, \alpha, R)$. Let us define bilinear maps

$$
\begin{aligned}
& x \triangleleft m=x \succ R_{M}(m)+\lambda x \succ m \quad \text { and } \quad x \triangleright m=R(x) \succ m \\
& m \triangleleft x=m \prec R(x)+\lambda m \prec x \quad \text { and } \quad m \triangleright x=R_{M}(m) \prec x .
\end{aligned}
$$

Then, $(M, \triangleleft, \triangleright, \beta)$ is a bimodule over the Hom-dendriform algebra.

Corollary 4.4: Let $(M, \triangleleft, \triangleright, \prec, \succ, \beta)$ be a bimodule over the q-HomTridendriform algebra $(D, \dashv, \vdash,, \alpha)$. Let us define

$$
\begin{aligned}
& x \succ^{\prime} m=x \triangleleft m+x \triangleright m+q x \succ m, \\
& m \prec^{\prime} x=m \triangleleft x+m \triangleleft x+q m \prec x .
\end{aligned}
$$

Then, $\left(M, \prec^{\prime}, \succ^{\prime}, \beta\right)$ is a bimodule over the Hom-associative $\operatorname{algebra}\left(D,{ }^{*}, \alpha\right)$.

Remark 4.2: Whenever the Rota-Baxter relations are verified, in Lemma 4.3 (resp. Lemma 4.4) for $\triangleleft$, $\triangleright$ and (resp. $\prec$ and $\succ$ ), then so is for $\triangleleft^{\prime}$ and $\triangleright^{\prime}$ (resp. $\triangleleft$ and $\triangleright$ ).

\section{Further Discussion and Conclusions}

In this paper we give structure theorems of Rota-Baxter algebras over q-Hom-tridendriform algebras and the corresponding bimodules, their twisting, and their connection with Hom-associative Rota-Baxter algebras and Hom-preLie algebras.

1. A similar analysis may be made for bimodules over flexible, admissible and alternative q-Hom-tridendriform Rota-Baxter algebras. Also, the procedure to twist classical algebraic structures to obtain the Hom-version may be applied to Rota-Baxter opera-tor on HomLeibniz algebras and L-Hom-tridendriform algebra.

2. Thanks to Theorem 3.2 one may think of the classification of q-Hom-tridendriform Rota-Baxter algebras of weight 0 and 1 (and the corresponding modules). But due to the size and the non-linearity of system (35)-(45), we hope to return to these questions elswhere.

3. One of the issue is to establish graded case (and the corresponding modules) of the following commutative diagram

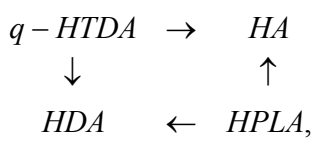

where we denote by HTDA: the category of Hom-tridendriform algebras, HARB: the category of Hom-associative Rota-Baxter algebras, HLRBA: the category of Hom-Lie Rota-Baxter algebras and HPLA: the category of Hom-Post-Lie algebras.

\section{References}

1. Baxter G (1960) An analytic problem whose solution follows from a simple algebraic identity. Pacific J Math 10: 731-742.

2. Rota GC (1969) Baxter algebras and combinatorial identities I, II. Bull Amer Math Soc 75: 325-329 Bull. Amer Math Soc 75: 330-334

3. Rota GC (1995) Baxter operators an introduction In Gian-Carlo Rota on combinatorics Contemp Mathematicians. Birkhauser Boston, Boston, MA 504512.

4. Connes A, Kreimer D (1998) Hopf algebras Renormalization and Noncommutative Geometry. Comm in Math Phys 199: 203.

5. Belavin AA (1982) Drinfeld VG, Solutions of the classical Yang-Baxter equation for simple Lie algebras. Funct Anal :159-180.

6. Semenov-Tian-Shansky MA (1983) What is a classical r-matrix? Funct Ana Appl 17: 259-272.

7. Szablikowski BM, Classical r-matrix like approach to Frobenius manifolds WDVV equations and flat metrics. to appear in $\mathrm{J}$ Phys $\mathrm{A}$.

8. Loday JL (2001) Dialgebras in Lecture Notes in Math, Springer Berlin 1763: 7-66.

9. Ebrahimi-Fard K (2002) Loday type Algebras and the Rota-Baxter Relation Lett Math Phys 61: 139-147.

10. Ebrahimi-Fard K (2008) Rota-Baxter algebras and dendriform algebras. Jour Pure Appl Algebra 212: 320-339.

11. Burgunder E, Ronco M (2009) Tridendriform structure on combinatorial Hopf algebras.

12. Makhlouf A, Silvestrov S (2010) Notes on Formal Deformations of Homassociative algebras and Hom-Lie algebras. Forum Math 22: 715-739.

13. Ammar F, Mabrouk S, Makhlouf A (2011) Quadratic n-ary Hom-Nambu algebras.

14. Aguiar M (2002) Infinitesimal bialgebras preLie and dendriform algebras.

15. Yau D (2012) Hom-Malcev Hom-alternative and Hom-Jordan algebras Int. Elect. Journ of Alg 11: 177-217.

16. Bakayoko I (2014) L-modules, L-comodules and Hom-Lie quasi-bialgebras. African Diaspora Journal of Mathematics 17: 49-64.

17. Bakayoko I (2014) Laplacian of Hom-Lie quasi-bialgebras. Inter Journ of Alg 8: 713-727.

18. Elhamdadi M, Makhlouf A (2010) Deformations of Hom-Alternative and HomMalcev algebras. To appear in Group Algebra and Geometries.

19. Abdaoui K, Ammar F, Makhlouf A (2013) Constructions and cohomology of generalized Hom-Lie algebras.

20. Bakayoko I, Oh SQ. Color Hom-Lie bialgebras and color Hom-Poisson bialgebras.

21. Bakayoko I (2014) Modules over color Hom-Poisson algebras J Gen Lie Theory Appl 8: 1.

22. Bakayoko I, Diallo OW (2015) Some generalized Hom-algebra structures. J Generalized Lie Theory Appl 9: 226 
23. Makhlouf A, Silvestrov S (2008) Hom-algebra structures. J Gen Lie Theory Appl 2: 51-64.

24. Ammar F, Ejbehi Z, Makhlouf A (2011) Cohomology and Deformations Homalgebras. Journal of Lie Theory 21: 813-836.

25. Makhlouf A, Yau D (2011) Rota-Baxter Hom-Lie-admissible algebras.

26. Hartwig J, Larsson D, Silvestrov S (2006) Deformations of Lie algebras using derivations. J Algebra 295: 314-361.
27. Yau D (2009) Hom-algebras and Homology. J Lie Theory 19: 409-421.

28. Donald $Y(2008)$ Module Hom-algebras.

29. Ammar F, Makhlouf A (2010) Hom-Lie algebras and Hom-Lie admissible super algebras. J Algebra 324: 1513-1528.

30. Donald $Y(2010)$ non-commutative Hom-Poisson algebras.

31. Humphreys JE (1972) Introduction to Lie algebra and representation theory Springer-Verlag New York Inc. 